“C 2017 IEEE. Personal use of this material is permitted. Permission from IEEE must be obtained for all other uses, in any current or future media, including reprinting/republishing this material for advertising or promotional purposes, creating new collective works, for resale or redistribution to servers or lists, or reuse of any copyrighted component of this work in other works." 


\title{
Design of a novel high frequency 2-D magnetic tester with nanocrystalline material
}

\author{
AngxuanLi ${ }^{1}$, Yongjian $\mathrm{Li}^{1 *}$, Changgeng Zhang ${ }^{1}$, Qingxin Yang ${ }^{1,2}$, and Jianguo Zhu ${ }^{3}$ \\ ${ }^{1}$ Province-Ministry Joint Key Laboratory of EFEAR, Hebei University of Technology, Tianjin, 300130, China, \\ ${ }^{2}$ Tianjin Key Laboratory of ATEEE, Tianjin Polytechnic University, Tianjin, 300387, China, \\ ${ }^{3}$ School of Electrical, Mechanical and Mechatronic Systems, University of Technology, Sydney, NSW 2007, Australia \\ Email:liyongjian@hebut.edu.cn
}

\begin{abstract}
The rotating magnetic properties of nanocrystalline materials at high frequencies play an important role in design and optimization of high frequency transformers with large capacity and high performance. In this paper, a novel high frequency rotating magnetic properties tester is designed, which is applied to measure the high frequency rotating magnetic properties of nanocrystalline materials. By changing the shape of the core, a new magnetic circuit structure is designed, which reduces the difficulty in assembly of the magnetic cores and improves the stability of the test system. A layer winding with copper foil is designed to ensure the accurate simulation of the high frequency rotating magnetic field. The influence of sample size and winding position on excitation effect is analyzed by finite element simulation. The magnetic circuit structure is optimized and the feasibility of the scheme is verified.
\end{abstract}

Index Terms-nanocrystalline, high frequency, new 2-D magnetic tester, winding with foil.

\section{INTRODUCTION}

With the extensive use of magnetic materials in electrical equipment, simulation and obtain the magnetic properties of materials in electrical equipment under the condition of actual operation has important significance to design and improve the performance of motor or transformer [1]. At present, the development of one dimensional magnetic properties tester is mature, and related international standards have been drawn up [2]. Two dimensional (2D) magnetic properties have been studied for more than 100 years, and the tester always been updated. The magnetic structure with four poles is designed by Germany Rheinisch-Westfaelische Technische Hochschule Aachen University. Japanese scholar Enokizono proposed a two-dimensional magnetic properties measurement device principle diagram, which can measure the magnetic properties of electrical steel in sheet [3]. Shenyang University of Technology produced a modified two-dimensional magnetic properties measurement tester, and studied the rotating magnetic properties of silicon steel lamination [4]. But with the development and application of high frequency transformer with large capacity, the magnetic characteristics of materials in high frequency become more

This work was supported in part by the Key Project of National Nat ural Science Foundation of China, under Grant 51237005, the National Key Basic Research Program of China (973 Project) under Grant 2015C B251000, National Key R \& D Program of China (2017YFB0903904), State Key Laboratory of Alternate Electrical Power System with Renewable Energy Sources, under GrantLAPS16002. and more important [5]. In the existing 2D magnetic properties tester, magnetic circuit structure is complex and the fabrication of magnetic core is difficult. The test result is easy to be affected by the assembly precision of the magnetic core. The excitation frequency of the tester system is low because the limited of core material and magnetic circuit structure.

In view of the above shortcomings, a novel type magnetic properties tester is designed, which provides a feasible scheme for obtaining high frequency rotating magnetic properties of nanocrystalline materials.

\section{THE NOVEL 2-D MAGNETIC PROPERTIES TESTER}

As shown in Fig 1, the magnetic measurement system includes: the main tester composed of core structures, which aim to finish the excitation and output the signal; the power amplifier to drive axial excitation and ensure the sample is fully magnetized; the multi-channel differential amplifying circuit, which realizes the amplification processing of the measured small induction signals; to produce the excitation signals and the collection of magnetic flux density (B) and magnetic field strength $(\mathrm{H})$ signals by the digital signal processing unit. The main tester is the key part of the whole system, mainly composed of magnetic cores, excitation windings and sensing coils $[6]$.

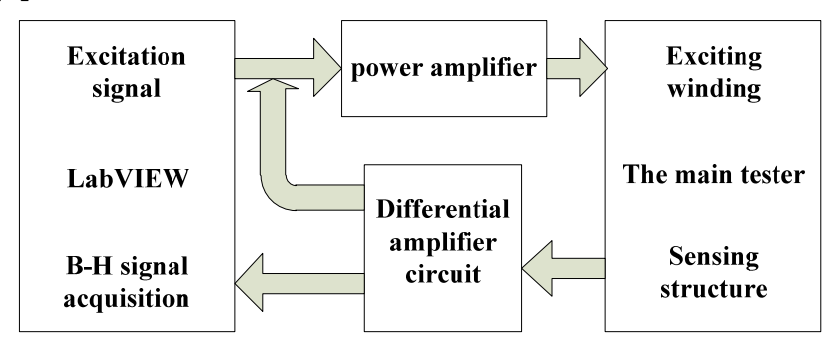

Fig. 1. Diagram of magnetic properties system .

\section{A. The Novel Tester}

As shown in Fig. 2(a), the double $\mathrm{C}$ ring vertical structure, which is the latest generation of $2 \mathrm{D}$ magnetic measurement device. In the device, the $\mathrm{C}$ type cores made of silicon steel lamination are formed into two independent circuits with the laminated or blocky sample, avoiding the effects between the magnetic circuits. The exciting windings are fixed on the two magnetic cores and the poles are processed into a wedge to achieve good effects of magnetism gathering, ensuring the sample is magnetized 
fully. The $B-H$ sensor is attached to the sample surface to collect voltage signals, which is sent back to the digital processing unit after differential amplification. The $B-H$ sensor is attached to the sample surface to collect voltage signals, which is sent back to the digital processing unit after differential amplification. Compared with the previous generation device, the magnetic circuit of the device is independent and the excitation process does not interfere with each other, improving the tester accuracy [7].

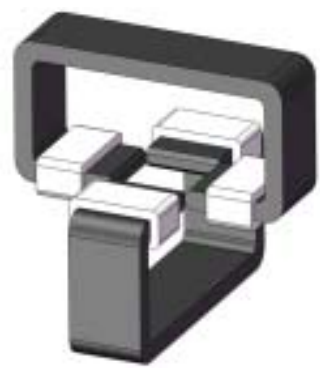

(a)

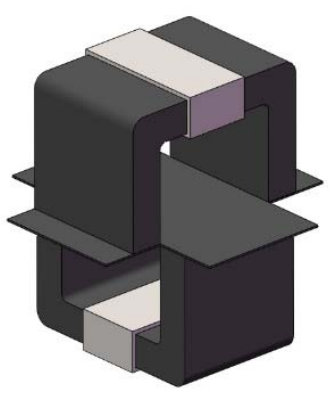

(b)
Fig. 2. Comparison diagram of magnetic circuit structure

When the frequency increases to $1 \mathrm{kHz}$, the heat loss of the magnetic core increases sharply, and the establishment of the high frequency magnetic field becomes very complex, so it is necessary to choose new material to make the magnetic core. Nanocrystalline materials with high permeability and the loss is only $1 / 10$ of silicon steel in high frequency [8].

The new magnetic circuit structure presented in this paper is shown in Figure 2(b), the two $U$ type cores are wound by high performance nanocrystalline ribbons to ensure that the core losses and the heating of excitation in a reasonable range under high frequency. Each core and sample form a magnetic circuit separately without affecting each other. In the two mutually perpendicular magnetic circuits, a rotating magnetic field is formed in the middle of the sample by controlling the phase difference of the applied voltage signal, and the $B-H$ sensor is placed in the center of the sample to collect signals. The magnetic circuit has the advantages of simple structure and easy processing of the pole that overcomes the difficulty of processing because the low mechanical strength of the nanocrystalline material, ensuring the feasibility of the device manufacture. The planar pole and the sample are directly contacted to form a magnetic circuit, eliminating the influence caused by the malposition of the pole effectively. The position of the exciting winding is transferred from the pole to the center of the magnetic core to avoid the interference between different exciting windings. The magnetic field vector of the central region of the sample is calculated as follows:

$$
\begin{aligned}
& B=\sqrt{B^{2}{ }_{x}+B^{2} y} \\
& \theta_{B}=\arctan \left(\frac{B_{y}}{B_{x}}\right)
\end{aligned}
$$

The uniformity of the magnetization in the center of the sample has an important influence on the measurement results for the $B-H$ sensor is placed in the center of the sample to collect signals.

\section{B. Simulation Analysis}

In the vertical structure of the double $\mathrm{C}$ ring, the $H$ of each magnetic circuit is measured by sensing coil in the center of the sample face. The processing error of wedge pole is large due to low mechanical strength of nanocrystalline material, which causes two poles not symmetry exactly. The offset of poles will cause a greater impact on the acquisition of $H$ signals when the magnetic cores are installed or removed. The finite element simulation(FEA) as shown in Figure 3, it can be seen that the magnetic field in the center of sample is not uniform due to the pole offset and process error, resulting in inaccurate testing.

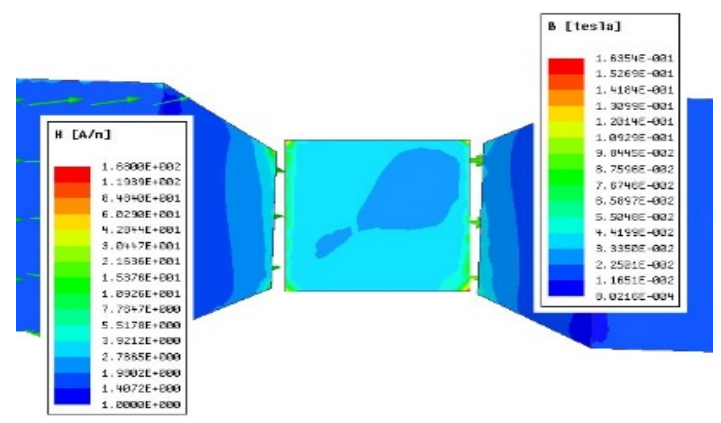

Fig. 3. The distribution diagram of magnetic induction strength and magnetic field intensity vector in poles and sample under pole offset.

In the novel magnetic circuit structure, two cores and sample are in contact so that the flux goes into the sample from the plane pole directly, producing a uniform magnetic field in the $100 * 100$ region of the sample for the $B-H$ sensor to collect singles. Without affecting the establishment of a rotating magnetic field, the assembly error is eliminated by simplifying the magnetic circuit structure and the measurement accuracy is improved by enlarging the uniform magnetic field area on the sample. The simulation of novel magnetic circuit structure and the uniformity of magnetic field in sample are shown in Fig.4.

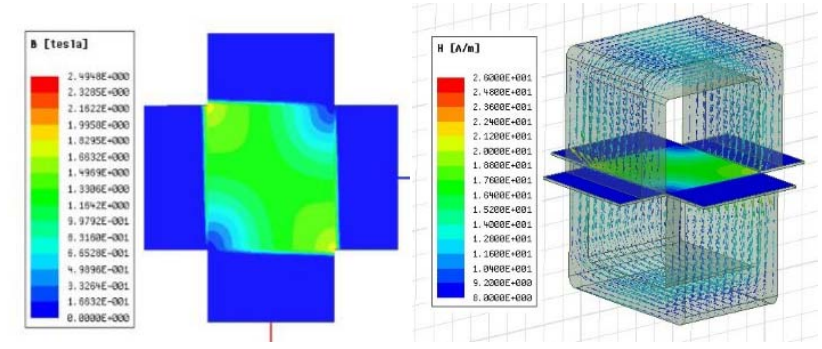

Fig. 4. Diagram of magnetic field intensity vector in core structure and magnetic induction strength in sample.

Reducing the mutual interference between magnetic circuits and ensuring the independence of each other is the key factor to obtain the uniform magnetic field in the novel circuit structure. The sample is designed as a square sheet of $200 * 200$ initially. Most of the flux forms a magnetic circuit along the core and the sample after the excitation 
winding excited the magnetic field. However, there are some magnetic flux passes through the sample into another core in the position near two poles, which not only affects the uniformity of magnetic field but also causes the local overheating and the increase of the loss. The shape of the sample is optimized to solve this problem by cutting the square of the $50 * 50$ at the four corners of the sample. By blocking the flux between the poles, the flux overlap is eliminated and the mutual interference of the magnetic circuits is solved. The magnetic flux density distribution diagram and the magnetic field intensity vector diagram finite is shown in the Fig.5. It can be seen that the magnetic field cores and sample are more uniformity and the overheating problem is reduced by optimized shape of sample.
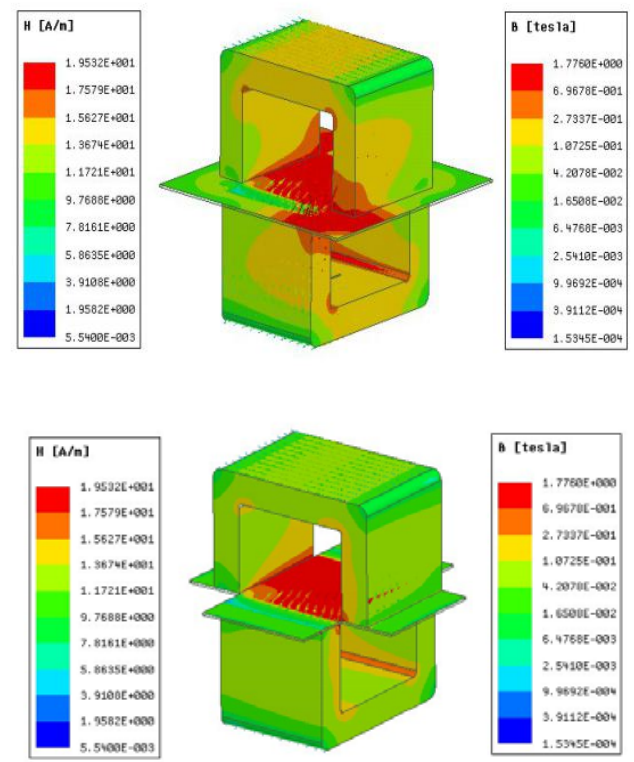

Fig. 5. Diagram of magnetic field intensity vector and magnetic induction strength in sample along with cores.

\section{Combined Sensing Structure}

The magnetic properties of materials magnetized by external magnetic field generally measured by the indirect method, which is to measure $H$ and $B$. The magnetization current method is not applicable to the measurement of two-dimensional or three-dimensional due to the uncertainty of the magnetic in the sample.

A sensing structure combining sensing coil and needle probe is designed, as shown in Fig. 6(a) and (b). Two $H$ sensing coils which are wound around a $0.4 \mathrm{~mm}$ thick epoxy resin board with 100 turns are attached on opposite sides of the sample to detect components of the vector $H$ in two directions. The basic principle of the $H$ sensing coils is that the magnetic field strength along tangential direction is continuous in the absence of current and electric field. The $H$ sensing coils should be attached as close as possible to the specimen surface in manufacture process to decrease the measurement error and improve the accuracy. According to Faraday's law of electromagnetic induction, vector $H$ can be calculated by:

$$
H(t)=\frac{1}{\mu_{0} K_{H}} \int_{0}^{t} V_{H}(t) d t
$$

where $\mu_{0}$ is the permeability of vacuum, $V_{H}$ is the inducted voltage of the $H$ sensing coil, $K_{H}$ is the coil coefficients of $H$ sensing coil which is calibrated in a long straight solenoid.

Wind the coil in the whole specimen will increase the length of gap between sample and cores and the material magnetic properties will be destroyed by drilling. The nondestructive needle probe method whose essence is one turn induction coil was proposed [9]. The vector B is measured by four needle probes which are equipped of the four corners of epoxy resin board. Compared with the sensing coil method, this method reduces the edge effect, uniform magnetic field of the testing region, and avoid punching in the specimen.

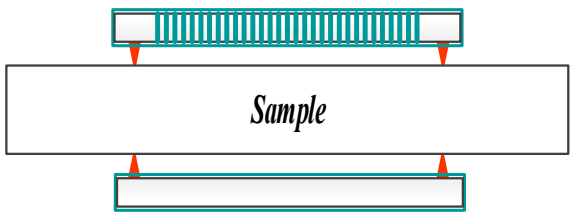

(a) front view

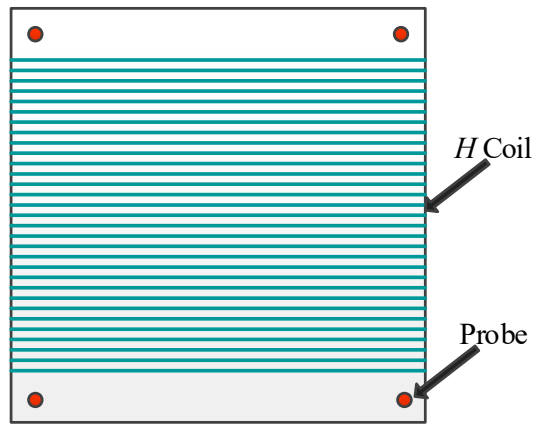

(b) overhead view

Fig. 6. Diagram of Combined sensing structure.

\section{Design of Excitation Structure}

The accurate simulation of magnetic field is the foundation and the most key part in the measurement of magnetic properties. The magnetic measurement system excites the magnetic field to magnetize the sample through the exciting winding. Considered the magnetic leakage generated by winding under high frequency, the optimum position of windings is determined by FEA based on the characteristics of magnetic circuit structure. To make the simulation closer to the actual situation, the length of gap between the windings and cores is set to $1 \mathrm{~mm}$ and the gap between the cores and sample is set to $0.5 \mathrm{~mm}$. Two typical positions of winding on magnetic cores are determined to analyze by FEA. The exciting current in the diagram (a) is set to $40 \mathrm{~A}$ and the exciting current in figure (b) is set to $20 \mathrm{~A}$. 


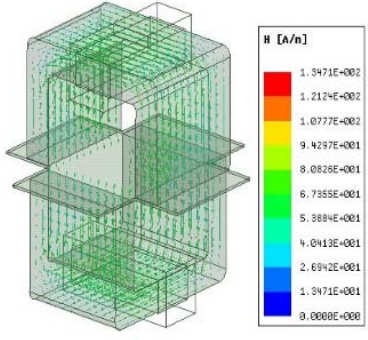

(a)

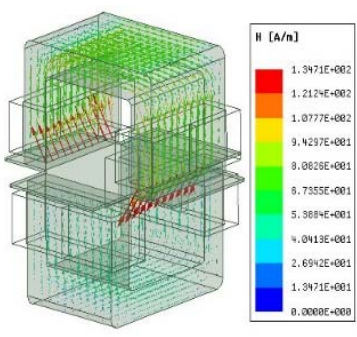

(b)
Fig.7. Diagram of magnetic field intensity vector in cores under different location of windings

It can be seen from the Fig. 7, the magnetic field intensity vector distribution is not uniform in $\mathrm{b}$ diagram and the magnetic field intensity near the windings is large due to the influence of leakage magnetic field in winding which not only increases the winding fever but also affects the distribution of magnetic induction intensity in sample. The magnetic flux distribution of the core in figure $b$ is uniform and the effects of leakage magnetic was eliminated. The effects of magnetic leakage generated are effectively eliminated by optimizing the location of the windings and the uniform of magnetic field distribution in sample is improving the accuracy of the device. The distribution diagram of magnetic induction strength in sample as shown in Fig.8.

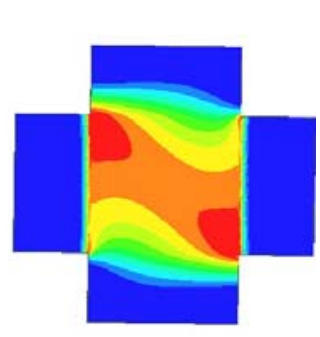

(a)

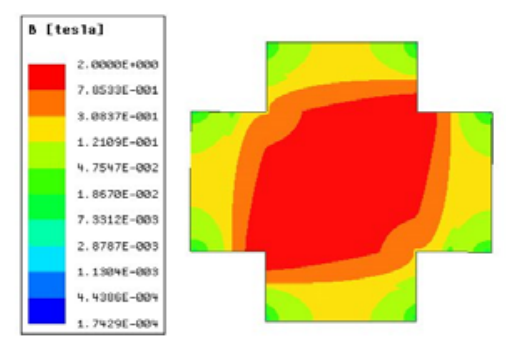

(b)
Fig.8. Diagram of magnetic induction strength distribution in sample under different location of winding

A flexible multi-layer excitation winding which can achieve magnetic properties measurement over wide range of excitation frequency was proposed by Reference [7]. The excitation winding is composed of three parts of coils with different layers, which can be connected in series or parallel corresponding to a certain frequency region. The high frequency effects are intensified and the loss of excitation winding becomes larger with the increase of frequency, so does the distribution parameters of winding. Compared to the cylindrical wire, the winding with foil has simple production process, compact structure and small magnetic leakage advantage. A layer winding with foil is designed in order to solve the high frequency effects and impedance matching of the excitation windings. The related formulas are as follows:

$$
L(i)=\frac{N^{2}}{R_{m}(i)}
$$

$$
\omega=2 \pi f
$$

$$
N I=H L
$$

Combining the above, the relation between the number of turns $(N)$ and the excitation frequency $(f)$ is:

$$
N=2 \pi f \sqrt{C R_{m}}
$$

When the excitation frequency is $50 \mathrm{kHz}$, the saturation field strength of nanocrystalline in cores is $180 \mathrm{~A} / \mathrm{m}$. Keep the magnetomotive force $F=N I=H L$ constant, the number of turns is proportional to the frequency of the excitation current. The winding with foil has sixteen layers which are divided into three coils to accommodate the winding parameters of the three frequency bands of the $2 \mathrm{~Hz}-1 \mathrm{kHz}, 1 \mathrm{kHz}-10 \mathrm{kHz}$ and 10 $\mathrm{kHz}-20 \mathrm{kHz}$. The width of copper foil in windings is $2 \mathrm{~cm}$ and the thickness is $0.5 \mathrm{~mm}$. The high frequency coil $(10$ $\mathrm{kHz}-20 \mathrm{kHz}$ ) has the first two layers and $15 \mathrm{kHz}$ is the typical frequency. The middle frequency coil $(1 \mathrm{kHz}-10$ $\mathrm{kHz}$ ) has middle six layers and $5 \mathrm{kHz}$ is the typical frequency. The third coil with outermost eight layers is connected with the high along with the middle frequency coils in series as the low frequency coil $(2-1 \mathrm{kHz})$ and 500 $\mathrm{Hz}$ is the typical frequency.

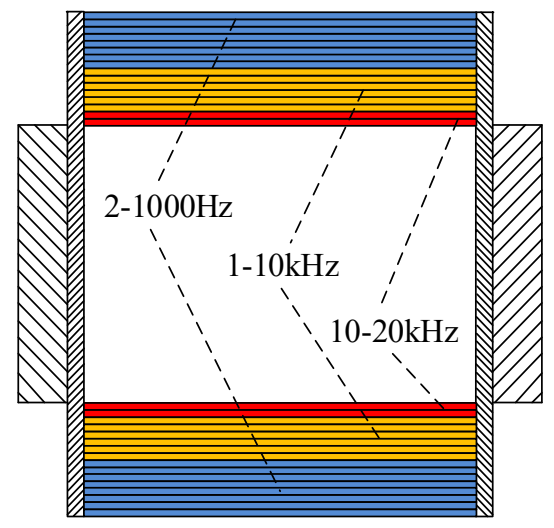

Fig. 9. Diagram of the layer winding with copper foil

\section{CONCLUSIONS}

This paper presents a novel 2D magnetic properties tester, which can study the magnetic characteristics of nanocrystalline in high frequency. Using high performance nanocrystalline materials to produce the cores, reducing the loss of magnetic circuit and the excitation frequency is increased to $50 \mathrm{kHz}$. By designing a new magnetic circuit structure and changing the shape of the cores, the difficulty of making and assembling magnetic cores is reduced effectively. The impact of processing and assembly inaccuracy on the experimental results are eliminated. The shape of sample and the position of exciting windings are optimized by the FEA, improving the measurement accuracy. A layered winding with foil is designed and the number of layers are calculated by the magnetic circuit equivalence along with series resonance principle to match different rang of exciting frequency, solving the problem of excitation in high frequency. 


\section{REFERENCE}

[1] R. Prochazka, J. Hlavacek, K. Draxler "Magnetic circuit of a high-voltage transformer up to $10 \mathrm{kHz}$," IEEE Trans. Magn., vol. 51, no. 1, pp. 1-4, January 2015.

[2] J. Sievert, H. Ahlers, M. Enokizono, S. Kauke, L. Rahf “The measurement of rotational power loss in electrical sheet steel using a vertical yoke system," J. Magn. Magn. Mater. 112(13): 91-94,1992.

[3] M. Enokizono "Magnetic hysteresis modelling for vector magnetic property," Int. J. Appl. Electromagn. Mech. 13(14): 203-208, 2001.

[4] Yanli Zhang, Young Hwan Eum, Dexin Xie, Chang Seop koh. "An improved engineering model of vector magnetic properties of grain-oriented electrical steels," et al. IEEE Trans Magn. 2008

[5] F. Blache, J. Keradec, B. Cogitore, "Stray capacitance of two winding transformers: Equivalent circuit measurements calculation and lowing," Proc. Conf. Rec. IEEE Ind Applicat. Soc. Annu. Meeting, vol. 2, pp. 1211-1217, 1994.

[6] Y. J. Li, J. G. Zhu, Q. X. Yang, et al. "Measurement of soft magnetic composite material using an improved 3-d tester with flexible excitation coils and novel sensing coils," IEEE Trans. Magn., vol. 46, no. 6, pp. 1971-1974, Jun. 2010.

[7] V. Goričan , A. Hamler, M. Jesenik , B. Štumberger ,M. Trlep "Measurement of magnetic properties of grainoriented silicon steel using round rotational single sheet tester (RRSST)," [J]. Journal of Magnetism and Magnetic Materials . 2004

[8] Makino A, Kubota T, Yubuta K, et al. "Low core losses and magnetic properties of Fe85-86Si1-2B8P4Cu1 nanocrystalline alloys with high $\mathrm{B}$ for power applications"[J]. Journal of Applied Physics, 109(7): 07A302. 2011,

[9] Q. X. Yang, Y. J. Li, Z. H. Zhao, et al. "Design of a 3-D rotational magnetic properties measurement structure for soft magnetic materials," IEEE Trans. Appl. Supercond., vol. 24, no. 3, pp. 1-4, 2014. 